Article

\title{
Computation of Stability Criterion for Fractional Shimizu-Morioka System Using Optimal Routh-Hurwitz Conditions
}

\author{
Yong Xian Ng and Chang Phang * (D) \\ Department of Mathematics and Statistics, Universiti Tun Hussein Onn Malaysia, Pagoh Campus, \\ Pagoh 84600, Malaysia; yongxianvmix@gmail.com \\ * Correspondence: pchang@uthm.edu.my; Tel.: +60-69742170
}

Received: 7 April 2019; Accepted: 22 April 2019; Published: 25 April 2019

check for updates

\begin{abstract}
Nowadays, the dynamics of non-integer order system or fractional modelling has become a widely studied topic due to the belief that the fractional system has hereditary properties. Hence, as part of understanding the dynamic behaviour, in this paper, we will perform the computation of stability criterion for a fractional Shimizu-Morioka system. Different from the existing stability analysis for a fractional dynamical system in literature, we apply the optimal Routh-Hurwitz conditions for this fractional Shimizu-Morioka system. Furthermore, we introduce the way to calculate the range of adjustable control parameter $\beta$ to obtain the stability criterion for fractional Shimizu-Morioka system. The result will be verified by using the predictor-corrector scheme to obtain the time series solution for the fractional Shimizu-Morioka system. The findings of this study can provide a better understanding of how adjustable control parameter $\beta$ influences the stability criterion for fractional Shimizu-Morioka system.
\end{abstract}

Keywords: Fractional Shimizu-Morioka System; stability criterion; Optimal Routh-Hurwitz conditions; time-fractional dynamical system

\section{Introduction}

This paper emphasizes the computation of stability criterion for a Shimizu-Morioka system in fractional order as shown below :

$$
\begin{aligned}
& x^{(\alpha)}(t)=y(t) \\
& y^{(\alpha)}(t)=(1-z(t)) x(t)-\sigma y(t) \\
& z^{(\alpha)}(t)=-\beta z(t)+(x(t))^{2}
\end{aligned}
$$

where $\sigma$ and $\beta$ are positive values. Here, we assume $\beta$ as the adjustable control parameter. The integer order system of Equation (1) was first proposed in [1] and has attracted the interest of researchers to study the stability and various types of bifurcation such as in [2-5]. However, the fractional order or arbitrary order of the system as in Equation (1) have received less attention; also see [6,7]. Unlike both [6,7], here, we apply optimal fractional order Routh-Hurwitz stability conditions which have recently been derived by Čermák and Nechvátal in [8]. Unlike the previous version of Routh-Hurwitz stability conditions for fractional systems (as derived in [9]), these new optimal Routh-Hurwitz condition serve as necessary and sufficient conditions to guarantee that all roots of the characteristic polynomial obtained from the linearization process are located inside the Matignon stability sector. Furthermore, the optimal Routh-Hurwitz conditions obtained were in an explicit form. The optimal Routh-Hurwitz conditions were successfully applied to study the fractional dynamical systems such as 
the fractional Lorenz system [8] and fractional Rössler System [10]. However, it is still yet to be applied for other fractional systems such as the fractional Shimizu-Morioka system.

On the other hand, the Shimizu-Morioka system can be considered as a simplified system for investigating the dynamic bahaviour of the well-known Lorenz system. However, due to its rich dynamic behaviour, and especially chaotic behaviour of its solutions, the Shimizu-Morioka system has its self-interest [2-5]. Unfortunately, there are not many published results regarding the stability and bifurcation analysis for the Shimizu-Morioka system in fractional orders. In this research direction, other fractional dynamical systems such as the fractional Lorenz system and many more always receive attention from researchers to study the dynamical behaviour of their stability analysis and bifurcation analysis [11-14]. This fractional order Shimizu-Morioka system can be considered as the generalized fractional Lorenz system which shows hyperchaotic and has important applications such as in secure the communication [15]. Apart from that, searching for robust stability is always the concern for researchers. For instance, as worked by [16] for fractional order control system with interval uncertain coefficients and a time-delay.

On top of that, an analytical approach for computation of stability analysis is always an important approach, especially for dynamical systems in fractional orders. The new optimal Routh-Hurwitz conditions allow us to do the analysis in explicit form. By applying the optimal Routh-Hurwitz conditions, for the first time, under the fractional derivative order $\alpha$, the range of $\beta$ and its influences towards the fractional Shimizu-Morioka system and whether it has locally stable equilibria will be identified. Although the Routh-Hurwitz conditions can be considered as an old topic, due to the recent development in fractional calculus, the study of Routh-Hurwitz conditions for fractional systems has emerged recently. Among that are a new Routh-type table for fractional system [17], Routh-Hurwitz-Liénard-Chipart Criteria [18], and a graphical approach to study stability analysis for incommensurate fractional-order systems [19].

The outline of the present paper is given as follows. Some basic concepts of Shimizu-Morioka system and its fractional counterpart is given in Section 2. Section 3 comprises of some basic concepts of the optimal Routh Hurwitz conditions for fractional system. Section 4 discusses the calculation of stability criterion for fractional Shimizu-Morioka system by using optimal Routh Hurwitz conditions. Numerical result by using a fractional predictor-corrector scheme for fractional Shimizu-Morioka system is given in Section 5 to verify the result obtained from the previous sections. Section 6 is the conclusion of the paper.

\section{Shimizu-Morioka System}

\subsection{Integer Order Shimizu-Morioka System}

The integer order Shimizu-Morioka system as shown in Equation (2).

$$
\begin{aligned}
& \dot{x}=y \\
& \dot{y}=(1-z) x-\sigma y \\
& \dot{z}=-\beta z+x^{2}
\end{aligned}
$$

where $\sigma$ and $\beta$ are positive values. Here, we assume $\beta$ as the adjustable control parameter. If $\beta>0$, then two equilibria appear, which are $E^{ \pm}=( \pm \sqrt{\beta}, 0,1)$. Then, we obtain the general characteristics equation of the system from the linearization along the equilibria.

$$
\lambda^{3}+(\beta+\sigma) \lambda^{2}+(\beta \sigma+z-1) \lambda+\beta(z-1)+2 x^{2}=0,
$$

and by substituting $E^{ \pm}$into Equation (3), we have the characteristics equation as follow,

$$
\lambda^{3}+(\beta+\sigma) \lambda^{2}+(\beta \sigma) \lambda+2 \beta=0 .
$$


From the standard Routh-Hurwitz conditions, it is easy to show that that all the $\lambda$ in the Equation (4) have negative real parts if and only if

$$
0<\beta<\beta^{*}=\frac{2-\sigma^{2}}{\sigma} .
$$

In other words, the integer order Shimizu-Morioka system is stable if and only if $0<\beta<\beta^{*}=\frac{2-\sigma^{2}}{\sigma}$.

\subsection{Fractional Order Shimizu-Morioka System}

Throughout this paper, we consider the standard fractional Shimizu-Morioka system as follows:

$$
\begin{aligned}
& { }_{0}^{C} D_{t}^{\alpha} x=y \\
& { }_{0}^{C} D_{t}^{\alpha} y=(1-z) x-\sigma y \\
& { }_{0}^{C} D_{t}^{\alpha} z=-\beta z+x^{2}
\end{aligned}
$$

where ${ }_{0}^{C} D_{t}^{\alpha}$ is the Caputo derivative operator defined as in Definition 1.

Definition 1. Let $\alpha>0, n=[\alpha]+1$ if $\alpha \notin \mathbb{N}, n=\alpha$ if $\alpha \in \mathbb{N}$ and $t>0$. The left Caputo fractional derivative of a function or order $\alpha$, denoted by ${ }_{0}^{C} \mathrm{D}_{t}^{\alpha} f(t)$ is

$$
{ }_{0}^{C} \mathrm{D}_{t}^{\alpha} f(t)=\frac{1}{\Gamma(n-\alpha)} \int_{0}^{t} \frac{f^{(n)}(\tau)}{(t-\tau)^{\alpha-n+1}} \mathrm{~d} \tau,
$$

with $n-1 \leq \alpha<n$

\section{Optimal Routh-Hurwitz Conditions for Fractional System}

In the classical theory of Routh-Hurwitz conditions, for three dimensional dynamical system, the characteristic polynomial in cubic form when $\alpha=1$ is as in Equation (8)

$$
P(\lambda ; a, b, c)=\lambda^{3}+a \lambda^{2}+b \lambda+c
$$

Let us consider $a, b, c$ are real coefficients. The Routh-Hurwitz conditions which for the characteristic polynomial in Equation (8) have all zeroes, i.e., $\lambda$ with negative real parts if and only if

$$
a>0, b>0 \text { and } 0<c<a b .
$$

By using the linearization theorem, we get that the equilibria, $E^{ \pm}=( \pm \sqrt{\beta}, 0,1)$ are locally asymptotically stable if all the zeroes $\lambda_{i}, i=1,2,3$ of Equation (4) are located inside the Magtinon stability sector [8].

$$
\left|\arg \left(\lambda_{i}\right)\right|>\frac{\alpha \pi}{2}
$$

Then, the Routh-Hurwitz conditions are only sufficient for validity of the Magtinon stability sector in Equation (10) with $0<\alpha<1$. Under this situation, the stability sector of the set of all triplets $(a, b, c)$ is wider and significantly more complicated due to Equation (10).

Follow the work in [8], we define $B L(\alpha)$ as the boundary locus as follow.

$$
B L(\alpha)=(a, b, c) \in\left(\mathbb{R}^{3}\right): \text { There exist } \lambda \in \mathbb{C},|\arg (\lambda)|=\frac{\alpha \pi}{2} \text { and } P(\lambda ; a, b, c)=0
$$

which $\lambda$ can be computed through $\lambda=\omega(\cos (\alpha \pi / 2)+i \sin (\alpha \pi / 2))$. Substitute the value of $\lambda$ into Equation (8), we obtain the following real and imaginary part. 


$$
\begin{array}{r}
4(\cos (\alpha \pi / 2))^{3} \omega^{3}+2(\cos (\alpha \pi / 2))^{2} a \omega^{2}-3 \cos (\alpha \pi / 2) \omega^{3}+\cos (\alpha \pi / 2) b \omega-a \omega^{2}+c=0 \\
4 \sin (\alpha \pi / 2)(\cos (\alpha \pi / 2))^{2} \omega^{3}+2 \sin (\alpha \pi / 2) \cos (\alpha \pi / 2) a \omega^{2}-\omega^{3} \sin (\alpha \pi / 2)+\sin (\alpha \pi / 2) b \omega=0
\end{array}
$$

Solving the above equations, we will get

$$
\begin{aligned}
& b=-\omega\left(4(\cos (\alpha \pi / 2))^{2} \omega+2 \cos (\alpha \pi / 2) a-\omega\right) \\
& c=2 \cos (\alpha \pi / 2) \omega^{3}+a \omega^{2}
\end{aligned}
$$

From the first equation of Equation (13), we get

$$
\omega=\frac{-\cos (\alpha \pi / 2) a \pm \sqrt{(\cos (\alpha \pi / 2))^{2} a^{2}-4(\cos (\alpha \pi / 2))^{2} b+b}}{4(\cos (\alpha \pi / 2))^{2}-1}
$$

and putting Equation (14) into second equation of Equation (13), and after some algebra manipulation, lead to

$$
c^{ \pm}(a, b ; \alpha)=\frac{-a b \pm 2 \gamma\left(a^{2}-4 b \gamma^{2}+b\right) \sqrt{a^{2} \gamma^{2}-4 b \gamma^{2}+b}+2 a \gamma^{2}\left(-a^{2}+4 b \gamma^{2}+b\right)}{\left(4 \gamma^{2}-1\right)^{3}}
$$

where $\gamma=\cos \left(\frac{\alpha \pi}{2}\right)$.

To further describe this set, we use the following theorem which derived in [8].

Theorem 1. Let $2 / 3<\alpha<1$. The Equation Equation (8) has all the zeroes $\lambda_{i}$ which satisfies the stability condition for fractional system as in Equation (10) if and only if any of the following conditions hold:

(i) $\quad a>0, b>0,0<c<c^{-}(a, b ; \alpha)$.

(ii) $\quad a>0, \hat{b} \leq b \leq 0, c^{+}(a, b ; \alpha)<c<c^{-}(a, b ; \alpha)$.

(iii) $\quad a \leq 0, b>\bar{b}(a ; \alpha), 0<c<c^{-}(a, b ; \alpha)$.

where $\bar{b}(a ; \alpha)=\frac{a^{2}}{4 \gamma^{2}}, \hat{b}(a ; \alpha)=\frac{a^{2} \gamma^{2}}{4 \gamma^{2}-1}$ and

$$
c^{ \pm}(a, b ; \alpha)=\frac{-a b \pm 2 \gamma\left(a^{2}-4 b \gamma^{2}+b\right) \sqrt{a^{2} \gamma^{2}-4 b \gamma^{2}+b}+2 a \gamma^{2}\left(-a^{2}+4 b \gamma^{2}+b\right)}{\left(4 \gamma^{2}-1\right)^{3}}
$$

with $\gamma=\cos \left(\frac{\alpha \pi}{2}\right)$.

Proof. The proof is following [8]. For $c \leq 0$, the zeros $\lambda_{i}$ of polynomial Equation (8) must has the positive real parts, where contradicts with the stability sector Equation (10). Thus, we define $H S(\alpha)$ as the boundary locus in half-space for $c$ is always positive.

$$
H S(\alpha)=\left\{(a, b, c) \in\left(\mathbb{R}^{2} \times \mathbb{R}^{+}\right): \text {There exist } \lambda \in \mathbb{C},|\arg (\lambda)|=\frac{\alpha \pi}{2} \text { and } P(\lambda)=0\right\}
$$

Let $2 / 3<\alpha<1$, we consider the $T S(\alpha)$ as the set of $(a, b, c) \in\left(\mathbb{R}^{2} \times \mathbb{R}^{+}\right)$for the conditions in Theorem 1 holds.

We denote $C S(\alpha)$ as the complement of the $(T S(\alpha) \cup H S(\alpha))$ in the half-space $\left(\mathbb{R}^{2} \times \mathbb{R}^{+}\right)$. Hence, we have the whole half-space with $(T S(\alpha) \cup H S(\alpha)) \cup C S(\alpha)$.

It is enough for us to check the Theorem 1 by assuming the triplets $(a, b, c)$ represent in $T S(\alpha)$ and $C S(\alpha)$ respectively. First, we assume $(a, b, c)$ as $(0.8,0.7,0.5)$ lying inside $T S(\alpha)$. By directly solving the polynomial Equation (8) equal to 0 , we get all the zeros $\lambda_{i}$ have negative real parts, where 
$\lambda_{1}=-0.7526, \lambda_{2,3}=-0.0237 \pm 0.8147 \mathrm{I}$ and $\left|\arg \left(\lambda_{1,2,3}\right)\right|>\frac{\alpha \pi}{2}$ is satisfied. Thus, the zeros $\lambda_{i}$ is satisfied the Magtinon stability sector Equation (10) when $(a, b, c) \in T S(\alpha)$.

Then, we assume $(a, b, c)$ as $(-0.8,-0.7,0.5)$ which lying inside $C S(\alpha)$. Similarly procedure, we obtain that the zeros $\lambda_{i}$ have positive real parts, where $\lambda_{1}=-0.8141, \lambda_{2}=0.6141$ and $\lambda_{3}=1$. Thus, we obviously see that the zeros have positive real parts. In addition, Equation (10) is not satisfied since $\left|\arg \left(\lambda_{2,3}\right)\right|=0$. Therefore, we can say that the zeros $\lambda_{i}$ is not satisfied Equation (10) when $(a, b, c) \in C S(\alpha)$. Conversely, we say that the zeros $\lambda_{i}$ of Equation (8) satisfy Equation (10) if and only if any of the conditions of Theorem 1 holds.

\section{Stability Analysis of the Fractional Shimizu-Morioka System}

In this section, we will compute the stability criterion for the fractional Shimizu-Morioka system as in Equation (6). From Section 2, we will limit our value of $\sigma$ in $[0, \sqrt{ } 2]$. From the calculation, the coefficients of characteristic polynomial as in Equation (8) for fractional system in Equation (6) are $a=\beta+\sigma>0, b=\beta \sigma>0$ and $c=2 \beta>0$. Thus we can analyze local asymptotic stability of the equilibria by applying fractional optimal Routh-Hurwitz conditions [8]. It is obvious that the system in Equation (6) are locally asymptotically stable for any $\beta>0$ if $0<\alpha \leq 2 / 3$. If $2 / 3<\alpha<1$, then Theorem 1(i) in Section 3 will be applied. In this case, since all the $a, b$ and $c$ for the characteristic polynomial are bigger than 0 , the only relevant condition for calculating the stability criterion is the inequality $c<c^{-}(a, b ; \alpha)$. Hence, we shall explain some basic steps of the computational procedures to obtain the stability criterion for this fractional Shimizu-Morioka system.

By substituting the $a=\beta+\sigma>0, b=\beta \sigma>0$ and $c=2 \beta>0$ of the fractional Shimizu-Morioka system into $c<c^{-}(a, b ; \alpha)$, we can get the inequality as follows

$$
A \beta^{3}+B \beta^{2}+C \beta+D>\left(E \beta^{2}+F \beta+G\right) \sqrt{H \beta^{2}+I \beta+J}
$$

where

$$
\begin{aligned}
& A=2 \cos ^{2}\left(\frac{\alpha \pi}{2}\right) \\
& B=\sigma\left[1+4 \cos ^{2}\left(\frac{\alpha \pi}{2}\right)-8 \cos ^{4}\left(\frac{\alpha \pi}{2}\right)\right] \\
& C=\sigma^{2}\left[1+4 \cos ^{2}\left(\frac{\alpha \pi}{2}\right)-8 \cos ^{4}\left(\frac{\alpha \pi}{2}\right)\right]+2\left[4 \cos ^{2}\left(\frac{\alpha \pi}{2}\right)-1\right]^{3} \\
& D=2 \sigma^{3} \cos ^{2}\left(\frac{\alpha \pi}{2}\right) \\
& E=-2 \cos \left(\frac{\alpha \pi}{2}\right) \\
& F=-2 \sigma \cos \left(\frac{\alpha \pi}{2}\right)\left[3-4 \cos ^{2}\left(\frac{\alpha \pi}{2}\right)\right] \\
& G=-2 \sigma^{2} \cos \left(\frac{\alpha \pi}{2}\right) \\
& H=\cos ^{2}\left(\frac{\alpha \pi}{2}\right) \\
& I=\sigma\left[1-2 \cos ^{2}\left(\frac{\alpha \pi}{2}\right)\right] \\
& J=\sigma^{2} \cos ^{2}\left(\frac{\alpha \pi}{2}\right)
\end{aligned}
$$

We should notice that when $\alpha$ is approaching to 1, the inequality in Equation (16) becomes Equation (5) actually. This can be said that this computation is the generalization of integer order system to fractional order or arbitrary order system. Now, we can analyze the inequality Equation (16) by squaring it and reducing it into polynomial, $Q(\beta)$. 


$$
\begin{aligned}
Q(\beta)= & \left(E^{2} H-A^{2}\right) \beta^{6}+\left(E^{2} I+2 E F H-2 A B\right) \beta^{5} \\
& +\left(E^{2} J+2 E F I+2 E G H+F^{2} H-2 A C-B^{2}\right) \beta^{4} \\
& +\left(2 E F J+2 E G I+F^{2} I+2 F G H-2 A D-2 B C\right) \beta^{3} \\
& +\left(2 E G J+F^{2} J+2 F G I+G^{2} H-2 B D-C^{2}\right) \beta^{2} \\
& +\left(2 F G J+G^{2} I-2 C D\right) \beta+G^{2} J-D^{2}
\end{aligned}
$$

If we fixed the value of $\sigma$, we can evaluate the dependence of abjustable control parameter $\beta$ on the $\alpha$, i.e., fractional derivative order in the Caputo sense. In this case, from the result in Section 2, we shall limit the $\sigma$ only from 0 to $\sqrt{ } 2$. Thus, we illustrate the fractional Shimizu-Morioka system as in Equation (6) with the value $\sigma=0.736$. We obtain the following fractional system.

$$
\begin{aligned}
& { }_{0}^{C} D_{t}^{\alpha} x=y \\
& { }_{0}^{C} D_{t}^{\alpha} y=(1-z) x-0.736 y \\
& { }_{0}^{C} D_{t}^{\alpha} z=-\beta z+x^{2}
\end{aligned}
$$

Thus we analyze Equation (19) with respect to the bifurcation parameter $\beta$. In the sequel, we have the inequality Equation (16) with

$$
\begin{aligned}
A & \equiv A(\alpha)=2 \cos ^{2}\left(\frac{\alpha \pi}{2}\right) \\
B & \equiv B(\alpha)=0.736+2.944 \cos ^{2}\left(\frac{\alpha \pi}{2}\right)-5.888 \cos ^{4}\left(\frac{\alpha \pi}{2}\right) \\
C & \equiv C(\alpha)=128 \cos ^{6}\left(\frac{\alpha \pi}{2}\right)-100.333568 \cos ^{4}\left(\frac{\alpha \pi}{2}\right)+26.166784 \cos ^{2}\left(\frac{\alpha \pi}{2}\right)-1.458304 \\
D & \equiv D(\alpha)=0.797376512 \cos ^{2}\left(\frac{\alpha \pi}{2}\right) \\
E & \equiv E(\alpha)=-2 \cos \left(\frac{\alpha \pi}{2}\right) \\
F & \equiv F(\alpha)=5.888 \cos ^{3}\left(\frac{\alpha \pi}{2}\right)-4.416 \cos \left(\frac{\alpha \pi}{2}\right) \\
G & \equiv G(\alpha)=-1.083392 \cos \left(\frac{\alpha \pi}{2}\right) \\
H & \equiv H(\alpha)=\cos ^{2}\left(\frac{\alpha \pi}{2}\right) \\
I & \equiv I(\alpha)=0.736-1.472 \cos ^{2}\left(\frac{\alpha \pi}{2}\right) \\
J & \equiv J(\alpha)=0.541696 \cos ^{2}\left(\frac{\alpha \pi}{2}\right)
\end{aligned}
$$

For these values $A(\alpha), \ldots, J(\alpha)$, from Equation (16), we let $A \beta^{3}+B \beta^{2}+C \beta+D=f(\beta ; \alpha)$ and $\left(E \beta^{2}+F \beta+G\right) \sqrt{H \beta^{2}+I \beta+J}=g(\beta ; \alpha)$. Thus, we analyze the inequality as shown in Equation (21).

$$
f(\beta ; \alpha)>g(\beta ; \alpha)
$$

where $\beta>0$ and $2 / 3<\alpha<1$. From the observation of the graph, it is obvious that $E(\alpha)<0, F(\alpha)<0$ and $G(\alpha)<0$ whenever $2 / 3<\alpha<1$. Hence, we have $g(\beta ; \alpha)<0$ for all $\beta>0$ and $2 / 3<\alpha<1$. Similarly, a direct calculation shows that $C(\alpha) \geq 0$ for any $2 / 3<\alpha \leq \alpha_{0}$, where

$$
\alpha_{0}=\frac{2}{\pi} \arccos (0.2747107675) \approx 0.8228357939
$$

Since $A(\alpha)>0, B(\alpha)>0$ and $D(\alpha)>0$ for any $2 / 3<\alpha<1$, Equation (21) is satisfied trivially for any $2 / 3<\alpha \leq \alpha_{0}$ and any $\beta>0$. Furthermore, $f(\beta ; \alpha) \geq 0$ for all $0<\beta \leq \beta^{*}=1.981391304$ and $2 / 3<\alpha<1$. Thus, Equation (21) is satisfied trivially for all $0<\beta<\beta^{*}$ and all $2 / 3<\alpha<1$. Hence, we assume $\alpha_{0}<\alpha<1$ and $\beta>\beta^{*}$ in our next analysis. 
Using elementary calculations based on the sign analysis of $\frac{d g}{d \beta}(\beta ; \alpha)$ and $\frac{d^{2} g}{d \beta^{2}}(\beta ; \alpha)$, one can check that, for any fixed $\alpha_{0}<\alpha<1, g(\beta ; \alpha)$ is decreasing in $(0, \infty)$.

$$
\frac{d g}{d \gamma}(\beta ; \gamma)=\frac{M(\beta ; \gamma)}{N(\beta ; \gamma)}
$$

where

$$
\begin{aligned}
M(\beta ; \gamma)= & 23.552 \beta^{3} \gamma^{4}-34.668544 \beta^{2} \gamma^{4}+12.75802419 \beta \gamma^{4}-4 \beta^{4} \gamma^{2}-2.944 \beta^{3} \gamma^{2} \\
& +21.66784 \beta^{2} \gamma^{2}-1.594753024 \beta \gamma^{2}-1.173738226 \gamma^{2}-1.472 \beta^{3} \\
& -3.250176 \beta^{2}-0.797376512 \beta
\end{aligned}
$$

and

$$
N(\beta ; \gamma)=\sqrt{\beta^{2} \gamma^{2}-1.472 \beta \gamma^{2}+0.541696 \gamma^{2}+0.736 \beta}
$$

The interval $\left(\alpha_{0}, 1\right)$ is bijectively mapped by $\gamma=\cos (\alpha \pi / 2)$ onto $\left(0, \gamma_{0}\right)$ where

$$
\gamma_{0}=\cos \left(\frac{\alpha_{0} \pi}{2}\right) \approx 0.2747107675
$$

Follow the similar procedure as in Section 4 of [8], we obtain

$$
\begin{gathered}
A(\alpha) \beta^{3}+B(\alpha) \beta^{2}+C(\alpha) \beta+D(\alpha) \\
=\left(E(\alpha) \beta^{2}+F(\alpha) \beta+G(\alpha)\right) \sqrt{H(\alpha) \beta^{2}+I(\alpha) \beta+J(\alpha)} \\
6 E(\alpha) H(\alpha) \beta^{3}+(5 E(\alpha) I(\alpha)+4 F(\alpha) H(\alpha)) \beta^{2} \\
+(4 E(\alpha) J(\alpha)+3 F(\alpha) I(\alpha)+2 G(\alpha) H(\alpha)) \beta+2 F(\alpha) J(\alpha)+G(\alpha) I(\alpha) \\
=2 \sqrt{\left(H(\alpha) \beta^{2}+I(\alpha) \beta+J(\alpha)\right)}\left(3 A(\alpha) \beta^{2}+2 B(\alpha) \beta+C(\alpha)\right)
\end{gathered}
$$

with unknowns $\beta$ and $\alpha$. By solving numerically the system formed by Equations (27) and (28), we will obtain a pair of $\alpha$ and $\beta$ in positive value which $\alpha$ lie inside $[2 / 3,1]$. Hence, we can conclude that the critical value for $\alpha$ is given as

$$
\alpha_{c r} \approx 0.9001093005
$$

Similar to [8], the geometrical analysis of Equation (16) yields the following conclusions.

Theorem 2. Let $0<\alpha<\alpha_{c r}$. Then the equilibria $E^{ \pm}$of Equation (19) are locally asymptotically stable for all $\beta>0$.

Theorem 3. Let $\alpha_{c r}<\alpha<1$. Then there exists a couple of values $\beta_{\alpha}^{*}<\beta_{\alpha}^{* *}$ (uniquely determined in the interval $\left(\beta^{*}, \infty\right)$ via Equation (27)) such that the equilibria $E^{ \pm}$of Equation (19) are locally asymptotically stable if either $0<\beta<\beta_{\alpha}^{*}$ or $\beta>\beta_{\alpha}^{* *}$. Moreover, $E^{ \pm}$are not stable whenever $\beta_{\alpha}^{*}<\beta<\beta_{\alpha}^{* *}$.

We will further explain the above theorems in following section.

\section{Numerical Results}

In this section, we will present some simulation of the stability analysis for the system Equation (1). For the simulation purpose, we applying generalized Adams-Bashforth-Moulton type predictor-corrector scheme for solving fractional differential equations developed in [20]. This numerical approach is widely used in the study of the dynamical behaviour of fractional dynamical system, such as in [21,22]. This is because most of the system of fractional differential equation or fractional dynamical system do not have analytical solution. Furthermore, we had also modified this approach for solving differential equation in Caputo-Fabrizio sense as in [23]. 
From the result in Section 4, when $\sigma=0.736$, we will obtain $\beta=0.1827310163$ and the critical value of $\alpha$ is 0.9001093006 . This condition is the fractional order Hopf bifurcation and where periodic solution should be occurred. Perturbate the adjustable control parameter $\beta$ which leads to the dissolve of limit cycle to unstable or stable condition. For the sake of simplicity when doing the simulation, we take $\alpha=0.9, \beta=0.1827, \sigma=0.736$ and the initial condition as $x(0)=0.5, y(0)=0, z(0)=0.8$. Figure 1 shows the periodic solution for the time series solution while the phase portrait of the trajectories with the initial condition $[0.5,0,0.8]$ shows the limit cycle appeared.

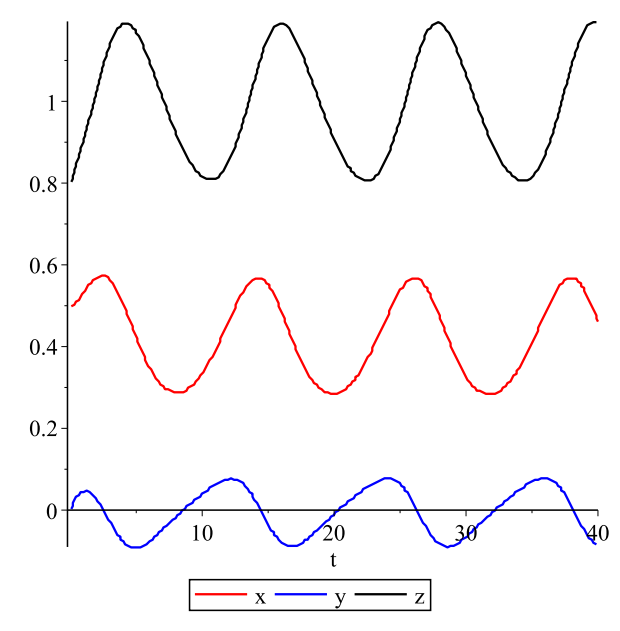

(a) Numerical solution show the periodic solution.

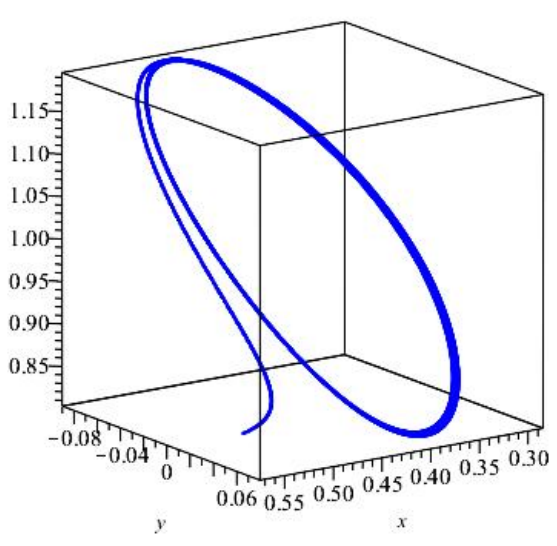

(b) Phase portrait show limit cycle.

Figure 1. Solution for system Equation (6) when $\alpha=0.9, \beta=0.1827, \sigma=0.736$.

When increasing the value of adjustable control parameter $\beta$, the solution becomes to stable toward the equilibrium $(\sqrt{\beta}, 0,1)$. We present the simulation result when $\beta=1$ as in Figure 2 .

On the other hand, based on Equation (16), the inequality is given as following.

$$
\begin{aligned}
& A(\alpha) \beta^{3}+B(\alpha) \beta^{2}+C(\alpha) \beta+D(\alpha) \\
& \quad>\left(E(\alpha) \beta^{2}+F(\alpha) \beta+G(\alpha)\right) \sqrt{H(\alpha) \beta^{2}+I(\alpha) \beta+J(\alpha)}
\end{aligned}
$$

We can reach the conclusion for the computation of stability criterion for fractional Shimizu-Morioka system by using optimal Routh-Hurwitz conditions as in Table 1 which follow Theorems 2 and 3.

Table 1. The stability condition for the system under certain range of $\beta$.

\begin{tabular}{ccc}
\hline$\alpha$ & Range of $\beta$ & Stability Condition \\
\hline$\alpha>\alpha_{c r}$ & Depend on Equation (30) & Stable \\
$\alpha<\alpha_{c r}$ & $0<\beta<\infty$ & stable \\
\hline
\end{tabular}

If $\alpha>\alpha_{c r}$, solving the inequality in Equation (30) will give the range of $\beta$ shows the fractional system is stable. As example, for the case when $\sigma=0.736$, where $\alpha_{c r}=0.9001093006$. If we let $\alpha=0.95$ which is $>\alpha_{c r}$, and we substitute it into Equation (30), we will be able to get the range of $\beta$ which give stability condition for the system. Following Theorem 3, we get $\beta^{*}=0.01114995609$ and $\beta^{* *}=1.025145474$. For other values of $\alpha$, the detail of calculations is shown in Table 2 . 


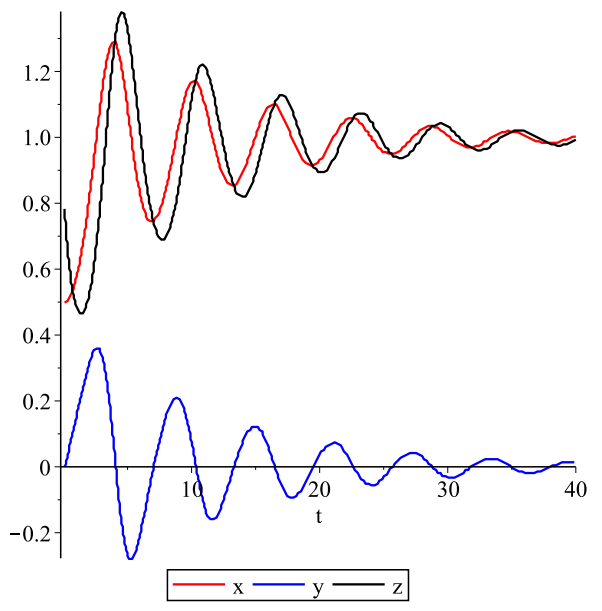

(a) Numerical solution show the stable solution.

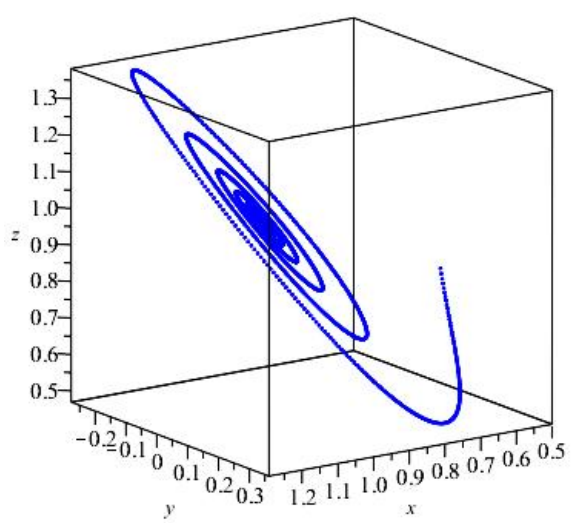

(b) Phase portrait show stable solution.

Figure 2. Solution for system Equation (6) when $\alpha=0.9, \beta=1, \sigma=0.736$.

Table 2. The stability condition for the system under certain range of $\beta$ when $\alpha_{c r}=0.9001093006$.

\begin{tabular}{ccc}
\hline $\boldsymbol{\alpha}$ & Range of $\boldsymbol{\beta}$ & Stability Condition \\
\hline $0.95>\alpha_{c r}$ & {$[0,0.01114995609],[1.025145474, \infty]$} & stable \\
$0.901>\alpha_{c r}$ & {$[0,0.1393510901],[0.2364214161, \infty]$} & stable \\
$0.89<\alpha_{c r}$ & {$[0, \infty]$} & stable \\
$0.85<\alpha_{c r}$ & {$[0, \infty]$} & stable \\
\hline
\end{tabular}

For illustration purpose, we take $\alpha=0.95$, the range of $\beta$ must be $[0,0.01114995609],[1.025145474, \infty]$ in order to get the stable solution. If the range of $\beta$ do not lie in that interval, the equlibria will be locally asymptotically not stable. We verified it through simulation for $\alpha=0.95, \beta=0.1827, \sigma=0.736$ as in Figure 3. All the calculations have done by using Maple. 


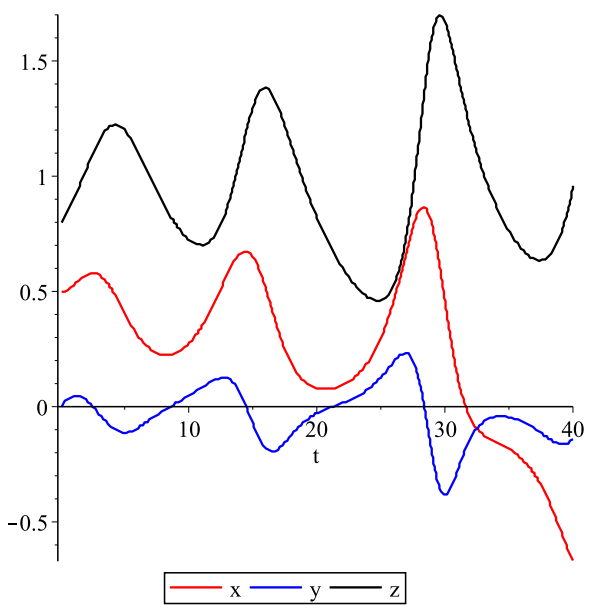

(a) Numerical solution show the unastable solution.

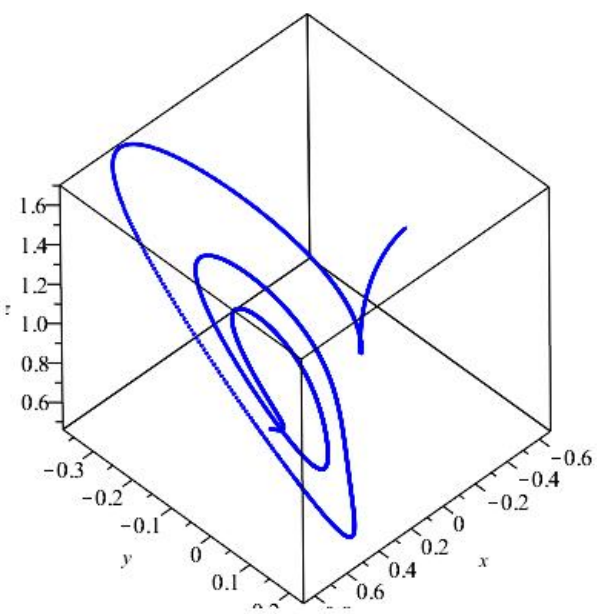

(b) Unstable solution.

Figure 3. Solution for system Equation (6) when $\alpha=0.95, \beta=0.1827, \sigma=0.736$.

\section{Conclusions}

In this paper, we have used new optimal Routh-Hurwitz conditions which also serve as necessary and sufficient conditions to compute the stability criterion for a fractional Shimizu-Morioka system. Here, we summarise our work as the following:

- For the $\sigma=[0, \sqrt{ } 2]$, the new optimal Routh-Hurwitz conditions enable us to detect the critical value of $\alpha$ for the stability criterion of fractional Shimizu-Morioka system when we use $\beta$ as control parameter.

- Furthermore, we introduce the way to calculate the range of adjustable control parameter $\beta$ to obtain the stability criterion for the fractional Shimizu-Morioka system.

The result was verified by the famous predictor-corrector scheme for fractional systems. The advantage of using this new optimal Routh-Hurwitz condition is that it enables us to obtain the range of $\beta$ which fulfills the stability criterion for the fractional Shimizu-Morioka system. However, lot of work need to be done by applying this new optimal Routh-Hurwitz condition to other fractional systems and this will probably be part of our future research work.

Author Contributions: Software, Y.X.N. and C.P.; Supervision, C.P.; Writing-original draft, Y.X.N.; Writing - review and editing, C.P.

Funding: This research was funded by UTHM through GPPS H049 and Ministry of Education Malaysia under the Fundamental Research Grant Scheme (FRGS) Vot K072 and the APC was funded by Fundamental Research Grant Scheme (FRGS) Vot K072.

Acknowledgments: The first writer wish to thank UTHM for the financial support during her study through GPPS H049. The second writer was supported by the Ministry of Education Malaysia under the Fundamental Research Grant Scheme (FRGS) Vot K072.

Conflicts of Interest: The authors declare no conflict of interest.

\section{References}

1. Shimizu, T.; Morioka, N. On the bifurcation of a symmetric limit cycle to an asymmetric one in a simple model. Phys. Lett. A 1980, 76, 201-204. [CrossRef]

2. Shil'nikov, A.L. On bifurcations of the Lorenz attractor in the Shimizu-Morioka model. Phys. D Nonlinear Phenom. 1993, 62, 338-346. [CrossRef]

3. Tigan, G.; Turaev, D. Analytical search for homoclinic bifurcations in the Shimizu-Morioka model. Phys. D Nonlinear Phenom. 2011, 240, 985-989.

4. Llibre, J.; Pessoa, C. The Hopf bifurcation in the Shimizu-Morioka system. Nonlinear Dyn. 2015, 79, $2197-2205$. [CrossRef] 
5. Capiński, M.J.; Turaev, D.; Zgliczyński, P. Computer assisted proof of the existence of the Lorenz attractor in the Shimizu-Morioka system. Nonlinearity 2018, 31, 5410. [CrossRef]

6. Akinlar, M.A.; Secer, A.; Bayram, M. Stability, synchronization control and numerical solution of fractional Shimizu-Morioka dynamical system. Appl. Math. Inf. Sci. 2014, 8, 1699. [CrossRef]

7. Danca, M.F.; Garrappa, R. Suppressing chaos in discontinuous systems of fractional order by active control. Appl. Math. Comput. 2015, 257, 89-102. [CrossRef]

8. Čermák, J.; Nechvátal, L. The Routh-Hurwitz conditions of fractional type in stability analysis of the Lorenz dynamical system. Nonlinear Dyn. 2017, 87, 939-954. [CrossRef]

9. Ahmed, E.; El-Sayed, A.M.A.; El-Saka, H.A. On some Routh-Hurwitz conditions for fractional order differential equations and their applications in Lorenz, Rössler, Chua and Chen systems. Phys. Lett. A 2006, 358, 1-4. [CrossRef]

10. Čermák, J.; Nechvátal, L. Local bifurcations and chaos in the fractional Rössler system. Int. J. Bifurc. Chaos 2018, 28, 1850098. [CrossRef]

11. Li, C.; Ma, Y. Fractional dynamical system and its linearization theorem. Nonlinear Dyn. 2013, 71, 621-633. [CrossRef]

12. Wang, Z.; Yang, D.; Ma, T.; Sun, N. Stability analysis for nonlinear fractional-order systems based on comparison principle. Nonlinear Dyn. 2014, 75, 387-402. [CrossRef]

13. Li, H.L.; Muhammadhaji, A.; Zhang, L.; Teng, Z. Stability analysis of a fractional-order predator-prey model incorporating a constant prey refuge and feedback control. Adv. Differ. Equ. 2018, 2018, 325. [CrossRef]

14. Li, H.; Cheng, J.; Li, H.B.; Zhong, S.M. Stability analysis of a fractional-order linear system described by the Caputo-Fabrizio derivative. Mathematics 2019, 7, 200. [CrossRef]

15. Wu, X.; Wang, H.; Lu, H. Modified generalized projective synchronization of a new fractional-order hyperchaotic system and its application to secure communication. Nonlinear Anal. Real World Appl. 2012, 13, 1441-1450. [CrossRef]

16. Gao, Z. Robust stability criterion for fractional-order systems with interval uncertain coefficients and a time-delay. ISA Trans. 2015, 58, 76-84. [CrossRef]

17. Liang, S.; Wang, S.G.; Wang, Y. Routh-type table test for zero distribution of polynomials with commensurate fractional and integer degrees. J. Frankl. Inst. 2017, 354, 83-104. [CrossRef]

18. Wiggers, S.L.; Pedersen, P. Routh-Hurwitz-Liénard-Chipart criteria. In Structural Stability and Vibration; Springer: Cham, Switzerland, 2018; pp. 133-140.

19. Shen, Y.; Wang, Y.; Yuan, N. A graphical approach for stability and robustness analysis in commensurate and incommensurate fractional-order systems. Asian J. Control 2019. [CrossRef]

20. Diethelm, K.; Ford, N.J.; Freed, A.D. A predictor-corrector approach for the numerical solution of fractional differential equations. Nonlinear Dyn. 2002, 29, 3-22. [CrossRef]

21. Abdullah, F.A.; Liu, F.; Burrage, P.; Burrage, K.; Li, T. Novel analytical and numerical techniques for fractional temporal SEIR measles model. Numer. Algorithms 2018, 79, 19-40. [CrossRef]

22. Moustafa, M.; Mohd, M.H.; Ismail, A.I.; Abdullah, F.A. Dynamical analysis of a fractional-order Rosenzweig-MacArthur model incorporating a prey refuge. Chaos Solitons Fractals 2018, 109, 1-13. [CrossRef]

23. Toh, Y.T.; Phang, C.; Loh, J.R. New predictor-corrector scheme for solving nonlinear differential equations with Caputo-Fabrizio operator. Math. Methods Appl. Sci. 2019, 42, 175-185. [CrossRef]

(C) 2019 by the authors. Licensee MDPI, Basel, Switzerland. This article is an open access article distributed under the terms and conditions of the Creative Commons Attribution (CC BY) license (http:/ / creativecommons.org/licenses/by/4.0/). 